\title{
EXISTING AND EVOLVING IN TWO MINDS: BELIEFS IN RELATION TO HEALTH AND ILLNESS EXPRESSED BY OLDER SOUTH AFRICANS
}

\author{
D.M. Bohman, PhD \\ Department of Health, Blekinge Institute of Technology, Sweden \\ N.C. van Wyk, PhD \\ Department of Nursing Science, University of Pretoria, South Africa \\ Corresponding author: neltjie.vanwyk@up.ac.za \\ S. Ekman, PhD \\ Department of Neurobiology, Care Sciences and Society, Karolinska Institutet, Swe- \\ den
}

\begin{abstract}
The aim of the study was to illuminate beliefs in relation to health and illness expressed by older Africans within the context of a society in transition, namely South Africa. An ethnographic research approach influenced by the interpretive phenomenological tradition was selected to gain an understanding of the participants' experiences. A focused ethnographic design was employed, using group and individual in-depth interviews and participant observations. Sixteen elderly persons (ten females and six males) from Hammanskraal, a rural area north of Pretoria, were involved in the research. The findings illuminate a world understanding where body and mind are inseparable and relationships provide the foundation for improving and maintaining health and being cured from illness. The ongoing transition that the elderly in South Africa experience influences health and illness beliefs, with a need to adapt to existing parallel health care systems, Western biomedicine and African traditional medicine. As the study draws attention to the importance of caring for the elderly to be contextualised, it is recommended that the care of the elderly be applied to the unique needs of the individual involved. Failure to do so may otherwise have severe consequences such as an apparent high risk of developing stereotypes, which can lead to cultural misunderstandings, prejudice and discrimination.
\end{abstract}

Keywords: aged, ethnographic research, health beliefs, phenomenology, South Africa

\section{INTRODUCTION}

Health and illness beliefs are essentially influenced by ethnic and cultural factors, both of which have significant implications for health care providers in encounters with patients. The process of interpreting symptoms and selecting who to approach for assistance, as 
well as routines and rituals for managing illness, are central aspects of the experience of illness and ongoing coping with chronic illnesses. Within this ethnographic study, the concept of culture refers to how meaning is constructed in a particular context, and ethnicity refers to a sense of belonging and group identity. The study demonstrates how a person inherits coping strategies as a member of a particular society. The strategies subsequently influence the individual's self and world understanding both on an emotional and interactive level (Helman, 2007:126).

\section{BACKGROUND TO THE STUDY}

An increasing number of studies are founded on the individual's personal experiences and beliefs regarding health and illness, which reveal that there are a variety of health and illness beliefs which exist within a particular society (Okello \& Neema, 2007:23). A study by Van Dyk (2001:9) provides an overview within an African context of the traditional African aspects of world perspectives and the importance of trying to understand the philosophical reasons behind beliefs regarding illness and which factors to take into account when caring for and counselling African people. According to Schatz and Gilbert (2012:23), despite a growing older population in sub-Saharan Africa, a limited number of studies have been conducted focusing on older people's health and illness beliefs. In South Africa, 60 to 80 percent of the African population frequently use the services of traditional healers (Puckree, Mkhize, Mgobhozi \& Lin, 2002:249) who play an essential role in the delivery of health care (Shizha \& Charema, 2011:170). Different categories of African traditional practitioners are represented in South Africa, including traditional healers, faith healers and diviners (Pretorius, 2001:253). Previous studies conducted by Bohman, Van Wyk and Ekman (2009:450), and Bohman, Vasuthevan, Van Wyk and Ekman (2007:335) focused on a group of older South Africans' experiences of everyday life and intergenerational relations and showed the changes in society due to its being in transition.

\section{PROBLEM STATEMENT}

The ongoing transition, articulated as moving with the times (Bohman et al, 2009:450), also influences various aspects of health and illness experience. According to Garenne (2007:67), Southern Africa is faced with a number of challenges, linked to the rapid modernisation of traditional African societies, presenting a number of social and health consequences. A recurrent theme is the influence of the HIV/Aids epidemic on the entire community and the personal lives of the elderly (Ogunmefun, Gilbert \& Schatz, 2011:99). Although there is an evident growing gap between generations, as bearers of traditional values, the older generation still plays a central role in providing financial support (Lloyd-Sherlock, Barrientos, Moller \& Saboia, 2012:250), as well as care to family members who are affected by the epidemic. HIV/Aids has had a devastating impact on 
people living in South Africa, with approximately 5.6 million people infected with HIV by 2009 (UNAIDS, 2012:4). Therefore, it is essential for health care professionals to enhance their understanding of and sensitivity towards individual beliefs in relation to health and illness during a time of transition.

\section{AIM OF THE STUDY}

The aim of this paper is to illuminate beliefs in relation to health and illness expressed by older Africans within the context of South Africa, a society in transition.

\section{DEFINITION OF CONCEPTS}

The concept of beliefs in this study comprises how individuals understand and interpret their experiences, not claiming a counter position between belief and knowledge.

The broader definition of health (World Health Organisation, 2006:5) emphasises not only the absence of disease, but also the presence of issues that relate to a sense of wellbeing.

Illness, in contrast to disease, represents personal, interpersonal and cultural reactions to disease or discomfort. Since illness is culturally constructed it is an intimate component of social systems, which includes rules for behaviour and cultural self-understanding (Helman, 2007:126).

The concept Africans in this study refers to black people from different ethnic origins who live in Africa.

\section{STUDY DESIGN}

For the purpose of this study, a focused ethnography design was employed, influenced by the interpretive phenomenological tradition, in accordance with the writings of Benner (1994:121) and Merleau-Ponty (1962:137) to gain an understanding of the participants' experiences, where the 'life world' constitutes common, immediate and lived experience and the individual provides the ultimate medium of experience and understanding of the phenomenal world.

\section{Study site}

The study was conducted in a semirural township in central South Africa close to Pretoria in the City of Tshwane Metropolitan Municipality. The area is underdeveloped, with insufficient infrastructure, a low level of housing and limited supply of education and 
health care. The site was selected because of its geographical location, approximately $50 \mathrm{~km}$ from Pretoria, but also because of ongoing collaboration with the Faculty of Health Sciences at the University of Pretoria. The local authorities were involved in information sessions with representatives of the community to explain the aim of the study. Following the initial meetings, older volunteers were invited to follow-up meetings held at a clinic in the area. As South Africa has limited population-based data (Tollman \& Kahn, 2007:6) and does not have detailed contact information on individual household members, information was disseminated with the assistance of health personnel at a local clinic in order to engage potential participants.

\section{Participants}

Participants were invited on the basis of the following inclusion criteria: (a) aged 55 and older; (b) equal number of women and men to obtain both genders' perspectives; (c) resident in the selected area; (d) proficiency in English; and (e) African. As one of the official languages, English is generally accepted by the South African population when interacting with people of different ethnic origins. Hence it was decided that English be the language used during the interviews. Africans were selected to participate, because they represent the majority group in the area and since the research was concentrated on the experiences of older Africans in general, and not on the experiences of only one African ethnic group.

Participants were selected consecutively and purposively from those who fulfilled the criteria and participated in the final selection meeting. The set-up criteria were initially adhered to, but had to be adjusted as a result of the majority of the participants being female. In order to achieve a more balanced perspective, the research team decided to increase the number of male participants; at a later stage a group of four older men from the area agreed to participate in the research. During the final selection, the research team decided to engage 16 older individuals in the study, including 10 females and 6 males (aged between 52 and 76 years).

\section{Data collection}

In line with the selected ethnographic approach (Flick, 2007:15) a variety of methods of data collection provided the basis for the study. Group interviews, individual in-depth interviews, participant observations with field notes were used to achieve a thorough and as broad a description as possible of the participants' life and the context. In order to engage in a more in-depth understanding of the context data were gathered during a time frame of six years. 


\section{Interviews}

Group interviews were carried out to familiarise the participants to the interview situation and to encourage conversations related to health and illness. Participants were divided into two groups for the group interviews. Individual in-depth interviews were conducted with ten older participants who had expressed interest in individual interviews during the group sessions. The interviews had a conversational structure to encourage the participants to share their experiences and to avoid interruption as much as possible. An interview guide consisting of the following themes was used: what constitutes a healthy life, how to maintain health, reasoning in connection with illness and disease, and how to handle sickness/illness.

\section{Participant observations}

Participant observations were conducted twice a year in sessions of four weeks with the 'observer as participant' approach. The researchers carried out observations for limited periods, interacting with participants, but mainly acting as observers. The observations included home visits and participation in community activities, which were documented with field notes after each session.

\section{Ethical considerations}

Following consent from the community representatives, local health authorities and approval from the Faculty of Health Sciences Research Ethics Committee of the University of Pretoria, participants were provided with information about the study. To avoid any potential misunderstanding, written information as well as verbal explanations were offered in English. Verbal and written consent was received from participants.

\section{Data analysis}

Data were analysed using an ethnographic technique in the interpretive phenomenological tradition. From this point of view, the ethnographic method seeks to identify the subjective meaning people ascribe to events rather than the 'objective' description of such events (Geertz, 2001:63). The analysis included several interrelated processes with a focus on the participants' own expressions related to health and illness. The verbatim-transcribed group interviews and individual interviews together with the field notes were read repeatedly to gain a general understanding of the text and its context. After the initial analysis, transcribed data were individually read and interpreted, followed by an interpretation of data as a whole in which the interpretation moved back and forth between the whole and parts to allow understanding, comparison and critical reflection on the text. The data were analysed for themes in relation to the participants' 
expressions of beliefs in relation to health and illness (thematic analysis), followed by a comparison of entire data and themes.

\section{Credibility of the findings}

In this ethnographic study containing a variety of data collection methods, the narratives from the interview sessions represent a powerful medium for gaining understanding about others by affording a context for insights into what one has not personally experienced. To establish credibility, in accordance with the ethnographic approach, sufficient time in the field was spent and the steps in the research process were made transparent and the participants' statements quoted (Lincoln \& Guba, 1985:300). As an ethnographic researcher, one's intention is to grasp the insider perspective, but it is as important to be self-aware about one's own role in the process and that one's interpretations are 'interpretations of interpretations', meaning that our own responses are culturally grounded, embedded in quite a different structure of aesthetic or emotional response than that of the members of the society described (Geertz, 2001:59).

\section{FINDINGS}

Four themes were identified, namely: (1) spirituality as an essential source for improving and maintaining health and being cured from illness; (2) the social body in relation to health and illness; (3) disruption of bodily knowledge; and (4) the interface between Western biomedicine and traditional African medicine.

\section{Spirituality as an essential source for improving and maintaining health and being cured from illness}

Within all of the narratives, to believe is to have religious beliefs or belief in a supernatural power. It was seen as essential and was considered the most important factor to improve or maintain health and to be cured from illness: 'It is a belief that goes at hope and then you focus yourself onto that. Some other people you know will even tell you; I'm cured because I believed in this type of thing and it cures.'

Spiritual belief played an essential role in relation to the participant's health and illness beliefs; God was consulted in the first instance regarding all issues. The body and mind were seen as a unity [body-mind] and through one's 'sensitive' body, one could connect with God. Illness was interpreted as a means to get in contact with God. There was a spiritual belief that being sick is normal; suffering and illness were seen as both natural and a way of purification. 'Sometimes there must be some hardship, it is the characteristics of life and you have periods of sickness to get to the next stage.' 
A fatalistic view was present in everyday actions, and if one fell sick it was considered 'God's will', if one remained healthy, it was also 'God's will': 'I thank God for the whole night he kept me. I think that is the thing that keeps me healthy. If not God's will is that you should not wake up in the morning.'

In the absence of illness, one tended to forget God, and falling ill was explained as God's will. What evolved is not a better person, but a better believer and illness must happen, as it was seen a part of normal life. Praying is the mediating tool for contact and through prayer one can interpret the signs of God. The day is divided into periods incorporating prayer, no matter if one is busy or not, or feeling unwell. To pray and sing has a positive impact on the body and makes all problems disappear: 'Singing, praying makes me very nice. I'm always happy in church. If you not feel well - nowadays I pray, several times per day.' To sing and move were seen to make one feel better. The pain is forgotten and good experiences from the past are remembered.

HIV/Aids was regarded as a new and modern disease, and the consequences of the disease could not be explained through their usual reasoning that disease makes a person stronger. On the contrary, the illness was seen as misfortune. HIV/Aids was interpreted as a modern disease that teaches little or nothing, but had the power of creating a curse if separation and the norms of contagion were ignored. In this way it was a disease with evolving meanings and cures that neither fully fit within biomedicine nor traditional African medicine, nor within a spiritual explanation of instruction and correction.

If my wife dies, I mustn't share a glass of water. And then if you done that, I've got bad luck and that bad luck turn to be something like HIV/AIDS. That is why most of the traditional healers they say there is no HIV/AIDS: it is this bad luck.

There was a belief that some traditional practitioners had a cure for HIV/Aids and that there were traditional practitioners who denied that HIV/Aids exists. Faith healers were regarded as an option to cure HIV/Aids. According to participants, through the belief in God and through prayer, one could be healed and cured in church. Although the church was seen as a place for salvation, there was still a fear of stigmatisation and reaction from the community when someone disclosed that he or she was infected with HIV/ Aids.

\section{The social body in relation to health and illness}

The impact of other people on one's health was a common theme, where the body was perceived as a part of a greater whole of relations. The participants were convinced that other people could cause illness, which made disturbed relationships an important reason for consulting traditional practitioners. The following observational field notes describe the practice of a traditional healer: 
She has items she uses during a consultation. The bone-throwing plays an important role in the treatment. First she throws the ... bones and shells on the mat and from the position of the different items she identifies a diagnosis. She also prepares Muti [mixture of herbs] to be used.

Traditional cutting and bone-throwing are actions conducted by the traditional healers that were seen to prevent illness caused by social interactions:

You know if someone provokes you or swear at you that can make you sick. In order for one to save himself, he or she has to go to a traditional healer for help. The traditional healer will cut you with a razor and apply some powder or medicine on the cuts he did on you to protect you.

Western medicine was not seen as an option for African relational diseases. Through the African healing process interviewees believed one could attain protection from evil spirits, but ultimately the protection depended on one's beliefs. The participants emphasised that if you did not believe, nothing would happen. Worries were related to feelings and concern for other people. One carried other people's problems in the heart and became physically and spiritually exhausted from this worry. The participants revealed that not only concern for others, but also personal moods affected the body, which could result in illnesses, such as high blood pressure or heart problems: 'Maybe you get sick because your heart is worrying all the time. Because of this worry I feel I will die. My heart beats so quickly.'

\section{Disruption of bodily knowledge}

Ageing was perceived by the participants as a process that influenced one's whole existence, including health and illness. The physical changes that occurred in the body were correlated to old age and explained as the body not being familiar anymore: 'When I was 60 to 70 years old it changed. When I go to the doctor I say why; they say it is old age. I don't know why but I don't like it.'

Age was seen as a period when one's strength declined, one had less energy, everything took longer, and the body was more susceptible to illnesses such as high blood pressure or diabetes. The female participants frequently related change in health with menopause, whilst the male participants' linked changes in health to how strongly they experienced their body and how physically fit they were. Old age was seen as a new chapter in life, unknown, uncertain and with new health issues, but nevertheless a natural phase that one could not escape. 


\section{The interface between Western biomedicine and traditional African medicine}

Existing between two paradigms (in this case, Western biomedicine and traditional African medicine), was repeatedly associated with help-seeking patterns. The participants' relationship with Western biomedicine was explained as seeking advice and treatment from doctors and nurses at the clinic, preferably for 'modern diseases' such as diabetes and high blood pressure. While acknowledging the competence of the health personnel, many of the participants did not necessarily follow all of the instructions they had received from the clinic. Their attitude towards medicine was expressed by one participant as: 'I can't say the doctor is not right. They are always right, but I didn't even touch the medicine.' There was a belief that if one started a treatment, the treatment would continue forever. When seeking advice and treatment in the field of traditional African medicine, the diversity of healers was evident.

Some of the participants expressed that they believed in traditional practitioners, but did not consult them because they had been influenced and persuaded by Western biomedicine.

To seek advice and treatment seems to be an interlocked action, where the participants moved between the two systems. They either consulted the nurses and doctors at the clinic or the traditional healer, depending on the situation. However, some diseases were thought to be untreatable at the clinic:

You see ... we've got sort of sickness which the doctors can't see immediately. Let me say if I got sores, maybe it is not just ordinary sores, but if I go to the hospital they are going to classify it as ordinary sores. But sometimes it is sores that are caused by somebody. Now the GP will not see that easily ... but if I got a flu I can't go to the healers. No, it is simple, I go to the doctor.

The participants could easily identify which illnesses and treatments they believed required traditional medicine. To put a curse on somebody or to be protected from a curse could only be handled by the traditional practitioner. The findings show patterns of who to approach in relation to health issues and also when to interact with either traditional African medicine or Western biomedicine.

\section{DISCUSSION}

The findings illuminate a group of older South Africans' responses to health and illness as they are in the interface between Western biomedicine and traditional African medicine. Spirituality, the importance of relationships and the social dimension of the body were seen as the most powerful sources in terms of preserving health and recovering from illness. 
As humans we live and relate to the surrounding world through our bodies, both consciously and unconsciously (Merleau-Ponty, 1962:137). Our understanding of the world is embodied in our bodily skills, and how we act and move can entail pieces of our understanding of self and world (Benner, 1994:20). According to Benner and Wrubel (1989:23), it is through a sensitive body that one experiences health and illness. The body is a unitary, integrated part of one's self (Orbach, 2009:7). The participants described how they sensed, communicated and alleviated suffering through their bodies.

The value of relationships in health and illness as demonstrated in this study comply with the notion of the embodied person existing through relationships (Merleau-Ponty, 1962:136). Helman (2007:23) claims that to a large extent, individuals embody the culture they live in and each human being has in a metaphorical sense two bodies; an individual body-self acquired at birth and a social body that is required in order to live in a particular society where cultural values shape the experience of the body. Subsequently, the social body provides each person with a framework for interpreting experiences. The participants in this study experienced bodily changes, which can be understood from a phenomenological perspective as their disrupted 'taken for granted life world' (Spichiger, Wallhagen \& Benner, 2005:308). The meanings of womanhood and manhood in old age influenced the participants' health-related and illness-related beliefs. Women predominately focus on bodily changes connected to menopause and men on declining physical strength (Santow, 1995:147). Bodily disruption in illness is perceived as normal, is spiritually explained and creates the potential for spiritual growth (Ming-Shium, 2006:1030).

The HIV/Aids epidemic did not correspond with the participants' belief that illness is normal and strengthens the spirit. On the contrary, it was referred to as a social circumstance of bad luck or misfortune, due to not complying with traditional rules. Such interpretation is common when an unexplained or untreatable illness occurs (Holland \& Hogg, 2010:20).

The findings elucidate the participants' relationships with two parallel systems of thought. In a time of transition, the influences of traditional African medicine are expressed in terms of continuing embedded relationships introduced by previous generations. Consultations with traditional African medicine are included. Likewise, the influence of Western biomedicine was presented in the classification of new diseases (diabetes and high blood pressure). This kind of assimilation of a more biomedicalinformed orientation can be explained as a part of an ongoing cultural transition (Leistner, 2003:20). The participants also express ambiguity towards the two systems in terms of avoiding traditional African medicine, based on the influences from institutions such as religious and educational institutions. But the participants did not trust the biomedicine to treat 'African' diseases and consequently preferred interventions from traditional healers. The intersection between collective and individual processes within 
societal transformation have been emphasised by Kleinman and Fitz-Henry (2007:52) and is consistent with the middle-range theory on experiencing transitions developed by Meleis et al (2000:14). Significant transitions have an effect on one's sense of self and how one relates to health and illness.

While traditional African medicine relies on ancestral connections and spiritual power to explain its efficiency (Van Wyk, 2009:12), Western biomedicine uses a mechanistic biomedical explanation for the occurrence of diseases (Lock \& Nguyen, 2010:82). Scientific theorising in biomedicine excludes the social, sentient embodied experiences of human beings as explanatory in disease causation. Acknowledging all progress linked to Western biomedical medicine, this study shows that even though people may accept Western biomedicine they preserve traditional, culturally influenced concepts to provide meaning to their health experience (Tabi \& Hodnicki, 2006:54). Traditional African medicine maintains its position as the first choice of health care for many Africans (Mulaudzi, 2009:32). Within an integrated health care system for Africa, nurses play an important role as mediators between patients making use of both Western biomedicine and traditional African medicine and health professionals trained in allopathic or Western biomedicine (Van Wyk, 2005:20).

\section{LIMITATION OF THE STUDY}

The study was conducted in one geographical area of South Africa and the authors thus do not claim that the findings can be generalised to all black people who live in the country.

\section{CONCLUSION}

This study provides a picture of a group of older South Africans' beliefs in relation to health and illness in a transitional cultural period, illuminating a view where body and mind are inseparable and where spirituality and relationships with others through the individual's 'social body' are central to improving and maintaining health. They experienced their bodies in old age as 'unfamiliar' and illness was sensed as bodily disruptions; interference in the everyday life experiences that were formerly merely taken for granted. Illness is not ultimately a negative sign, but is often rather experienced as an opportunity for growth for the individual. Furthermore, the findings demonstrate how the ongoing transition influences beliefs in relation to health and illness. Older South Africans sensitively navigate between the two curative and healing systems namely Western biomedicine and African traditional medicine. 


\section{RECOMMENDATIONS}

From a caring perspective the ethnographic phenomenological approach of this study is vital as it provided access to individual's sentient embodied life world. Although this study was conducted in a specific situation, the results draw attention to the importance of caring to be contextualised, the ability to adapt to ongoing transition on both a personal and social level, and to avoid the notion of totalising 'otherness' and cultural overgeneralisations. Failure to do so may have severe consequences, such as an apparent high risk of developing stereotypes, which can lead to cultural misunderstandings, prejudice and discrimination.

\section{ACKNOWLEDGEMENTS}

We would like to thank the participants for their contribution to the study and Dr Dorricah Peu, senior lecturer at the University of Pretoria for her support and assistance. We also wish to express our gratitude to Professor Patricia Benner, University of California, San Francisco for valuable feedback. The research was funded by grants from the Nordic Africa Institute, Uppsala, Sweden and Blekinge Institute of Technology, Sweden.

\section{REFERENCES}

Benner, P. 1994. Interpretive phenomenology: embodiment, caring, and ethics in health and illness. London: Sage.

Benner, P. \& Wrubel, J. 1989. The primacy of caring, stress and coping in health and illness. Menlo Park, CA: Addison-Wesley.

Bohman, D.M., Van Wyk N.C. \& Ekman, S.L. 2009. Tradition in transition: intergenerational relations with focus on the aged and their family members in a South African context. Scandinavian Journal of Caring Sciences, 23:446-455.

Bohman, D.M., Vasuthevan, S., Van Wyk, N.C. \& Ekman, S.L. 2007. 'We clean our houses, prepare for weddings and go to funerals': daily lives of elderly Africans in Majaneng, South Africa. Journal of Cross-Cultural Gerontology, 22:323-337.

Flick, U. 2007. Introduction: ethnography and participant observation. In M. Angrosino (ed), Doing ethnographic and observational research. London: Sage.

Garenne, M.L. 2007. Comment: health transitions and regressions in Southern Africa. Scandinavian Journal of Public Health, 35(Suppl 69):66-67.

Geertz, C. 2001. Thick description: toward an interpretive theory of culture. In R.M. Emerson (ed), Contemporary Field Research. Long Grove, IL: Waveland, 55-75.

Helman, C.G. 2007. Culture, health and illness. $5^{\text {th }}$ Edition. New York, NY: Oxford University Press.

Holland, K. \& Hogg, C. 2010. Cultural awareness in nursing and health care. $2^{\text {nd }}$ Edition. London: Arnold.

Kleinman, A. \& Fitz-Henry, E. 2007. The experiential basis of subjectivity: how individuals change in the context of societal transformation. In J. Biehl, B. Good \& A. Kleinman (eds), Subjectivity. Berkeley, CA: University of California Press. 
Leistner, E. 2003. African predicament: Africa weavering between past and modern time. Pretoria: Mlalo.

Lincoln, Y.S. \& Guba, E.G. 1985. Naturalistic inquiry. Newbury Park, CA: Sage.

Lloyd-Sherlock, P., Barrientos, A., Moller, V. \& Saboia, J. 2012. Pensions, poverty and wellbeing in later life: comparative research from South Africa and Brazil. Journal of Aging Studies, 26:243-252.

Lock, M.M. \& Nguyen, V.K. 2010. An anthropology of biomedicine. Chichester: John Wiley \& Sons.

Meleis, A.J., Sawyer, L.M., Im, E.O., Hilfinger, D.K., Messias, D.K. \& Schumacher, K. 2000. Experiencing transitions: an emerging middle-range theory. Advances in Nursing Science, 23(1):12-28.

Merleau-Ponty, M. 1962. Phenomenology of perception. New York, NY: Routledge.

Ming-Shium, T.U. 2006. Illness: an opportunity for spiritual growth. Journal of Alternative and Complementary Medicine, 12(10):1029-1033.

Mulaudzi, F.M. 2009. Traditional health practice. In N.C. van Wyk (ed), Integrative healthcare: a guide to meet the needs of Africa. Cape Town: Juta, 30-44.

Ogunmefun, C., Gilbert, L. \& Schatz, E. 2011. Older female caregivers and HIV/AIDS-related secondary stigma in rural South Africa. Journal of Cross-Cultural Gerontology, 26: 85-102.

Okello, E. \& Neema, S. 2007. Explanatory models and help-seeking behavior: pathways to psychiatric care among patients admitted for depression in Mulago Hospital, Kampala, Uganda. Qualitative Health Research,17(1):14-25.

Orbach, S. 2009. Bodies. London: Profile Books.

Pretorius, E. 2001. Traditional healers. In A. Ntuli (ed), South African Health Review. Pretoria: Health Systems Trust, 249-256.

Puckree, T., Mkhize, M., Mgobhozi, Z. \& Lin, J. 2002. African traditional healers: what health care professionals need to know. International Journal of Rehabilitation Research, 25:247-251.

Santow, G. 1995. Social roles and physical health: the case of female disadvantage in poor countries. Social Science \& Medicine, 40(2):147-161.

Schatz, E. \& Gilbert, L. 2012. 'My heart is painful': physical, mental and social wellbeing of older women at the times of HIV/AIDS in rural South Africa. Journal of Aging Studies, 26:16-25.

Shizha, E. \& Charema, J. 2011. Health and wellness in Southern Africa: incorporating indigenous and Western healing practices. International Journal of Psychology and Counselling, 3(9): 167-175.

Spichiger, E., Wallhagen, M.I. \& Benner, P. 2005. Nursing as a caring practice from a phenomenological perspective. Scandinavian Journal of Caring Sciences, 19:303-309.

Tabi, M.M. \& Hodnicki, D. 2006. Use of traditional healers and modern medicine in Ghana: International Council of Nurses. International Nursing Review, 53:52-58.

Tollman, S.M. \& Kahn, K. 2007. Health, population and social transition in rural South Africa. Scandinavian Journal of Public Health, 35(Suppl 69):4-7.

UNAIDS. 2012. South Africa HIV \& AIDS Statistics. http://www.unaids.org/en/regionscountries/ countries/southafrica/ (Accessed on 27 December 2012).

Van Dyk, A.C. 2001. 'Why me and not my neighbour?' HIV/AIDS care and counseling in a traditional African context. Curationis, 24(3):4-11.

Van Wyk, I.W.C. 2009. Sickness and healing in Africa: a religious perspective. In N.C. van Wyk (ed), Integrative healthcare: a guide to meet the needs of Africa. Cape Town: Juta, 12-29.

Van Wyk, N.C. 2005. Similarities in the meta-paradigm of nursing and traditional healing: an attempt to contribute to the integration of traditional medicine and Western medicine in Africa. Health SA Gesondheid, 10(3):14-22. 
World Health Organisation. 2006. Constitution of the World Health Organization. Basic documents, 45th Edition, Supplement, October 2006. http://www.who.int/governance/eb/ who_constitution_en.pdf (Accessed 27 January 2014). 